\title{
Hepatitis B virus X protein promotes epithelial-mesenchymal transition and metastasis in hepatocellular carcinoma cell line HCCLM3 by targeting HMGA2
}

\author{
YONG ZHA, QIAN YAO, JIN-SHENG LIU, YUAN-YUAN WANG and WEI-MING SUN \\ Department of Gastroenterological Surgery, The Third Affiliated Hospital of Kunming Medical University, \\ Kunming, Yunnan 650118, P.R. China
}

Received October 29, 2017; Accepted July 16, 2018

DOI: $10.3892 / \mathrm{ol} .2018 .9359$

\begin{abstract}
Chronic hepatitis B virus (HBV) infection is a major risk factor for hepatocellular carcinoma (HCC), and $\mathrm{HBV} \mathrm{X}$ protein $(\mathrm{HBx})$ serves an essential role in the development of HCC. However, its mechanism remains to be elucidated. The aim of the present study was to investigate the role and mechanism of the HBx protein in the epithelial-mesenchymal transition (EMT) and metastasis of HCC. The HCCLM3 cell line was transfected with a $\mathrm{HBx}$-expressing vector. The effects of $\mathrm{HBx}$ overexpression on proliferation, migration, invasion and EMT capacities of the HCCLM3 cell line were evaluated using MTT, migration and invasion assays, and western blotting, respectively. Furthermore, the impact of High mobility group AT-hook 2 (HMGA2) knockdown on HBx-mediated metastasis was investigated in the HCC cell line HCCLM3. The results demonstrated that HBx significantly upregulated HMGA2 expression, and enhanced the proliferation, EMT, invasion and migration in HCC cells. Furthermore, HMGA 2 knockdown almost abolished HBx-induced EMT and metastasis in HCC. The results of the present study suggest that $\mathrm{HBx}$ promotes the proliferation, EMT, invasion and migration of HCC cells by targeting HMGA2. HMGB2 may serve as a potential therapeutic target for $\mathrm{HBV}$-associated HCC.
\end{abstract}

\section{Introduction}

Hepatocellular carcinoma (HCC) is among the most lethal types of cancer, characterized by diagnosis at an advanced stage with regional vascular involvement, and distant metastasis (1).

Correspondence to: Professor Yong Zha, Department of Gastroenterological Surgery, The Third Affiliated Hospital of Kunming Medical University, 519 Kunzhou Road, Kunming, Yunnan 650118, P.R. China

E-mail: zhayong888@sina.com

Key words: HBV X protein, hepatocellular carcinoma, metastasis, high mobility group AT-hook 2
Hepatitis B virus (HBV) infection is one of the most common risk factors for HCC, particularly in Asia (2).

Hepatitis B virus $\mathrm{x}(\mathrm{HBx})$, a protein encoded by HBV virus, is essential for viral replication. Previous studies have demonstrated that $\mathrm{HBx}$ contributes to the progression of $\mathrm{HCC}$ by interacting with certain signal pathways, and promoting proliferation and invasive potential of HCC cells (3-6). HBx activates phosphatidylinositol 3-kinase (PI3K)/protein kinase B (AKT), the Notch signal pathway, and various transcription factors, including nuclear factor-kB $(\mathrm{NF}-\mathrm{kB})$ and activator protein 1 (AP-1) (3-5). However, the precise mechanisms by which $\mathrm{HBx}$ promotes the epithelial-to-mesenchymal transition (EMT) and metastasis of HCC remain unclear.

High mobility group AT-hook 2 (HMGA2), an architectural transcription factor, is overexpressed during embryogenesis, but not expressed in normal adult tissues $(7,8)$. Previous studies have reported HMGA2 is overexpressed in a variety of tumors, including HCC, breast cancer, non-small-cell lung cancer and gastric cancer (9-12). HMGA2 is involved in various essential biological processes, including DNA repair, apoptosis, cell proliferation, EMT and telomere restoration by regulating a wide range of gene expression levels in $\mathrm{HCC}$ (13-16). Given that HBx and HMGA2 serve important roles in HCC metastasis, the effects of HBx overexpression on EMT markers, invasion and metastasis of HCC cells were investigated in the present study. Furthermore, the role of HMGA2 in HBx-mediated HCC metastasis was explored.

\section{Materials and methods}

Cell culture. The HCCLM3 cell line was obtained from the Type Culture Collection of the Chinese Academy of Sciences (Shanghai, China). The cells were cultured in a humidified atmosphere of $5 \% \mathrm{CO}_{2}$ at $37^{\circ} \mathrm{C}$ in RPMI-1640 medium supplemented with $10 \%$ fetal bovine serum (FBS) (both from Hyclone; GE Healthcare Life Sciences, Logan, UT, USA).

HBx-expression vector construct and transfection. The construction of the HBx-expressing vector (pcDNA3.1-HBx) and control vector (pCDNA3.1-EGFP Vector) has been described in a previous study $(5,17)$. A full-length HBx gene was inserted into a pcDNA3.1 vector, and was identified using 
polymerase chain reaction (PCR), restriction endonuclease digestion and DNA sequencing methods. The PCR primers were as follows: HBx forward, 5'-CCGCTCGAGATGGCT GCTAGGCTGTGCTG-3' and reverse, 5'-CGGAATTCT TAGGCAGAGGTGAAAAAGTTG-3'. The PCR product was digested with $X h o I$ and $B g l I I$. The EGFP was inserted into a pcDNA3.1 vector between EcoRI and NotI as a control. The PCR primers for the control vector were as follows: CMV-f 5'-CGCAAATGGGCGGTAGGCGTG-3' and BGH-r 5'-TAGAAGGCACAGTCGAGG-3'. All ligated vectors were confirmed by DNA sequence analysis. HCCLM3 cells were transfected with the HBx-expressing vector, and then screened with puromycin to obtain stable cell clones. The stable HBX-expressing cells were termed HCCLM3-HBX cells.

RNA interference. The stable HBx-expressing HCCLM3 cell line was further assigned into two subgroups: $\mathrm{HBx}$ group and HMGA2 knockdown group [HBx + HMGA2 small interfering RNA (siRNA)]. The HMGA2 siRNA and corresponding control siRNA were designed and synthesized by Shanghai GenePharma Co., Ltd. (Shanghai, China). The HMGA2 siRNA target sequences was as follows: 5'-GGA AATGGCCACAACAAGTTG-3'. The cells were transfected using Lipofectamine 2000 (cat. no. 11668019; Invitrogen; Thermo Fisher Scientific, Inc, Waltham, MA, USA) according to the manufacturer's protocol. Briefly, the cells were seeded on 6-well plates at a density of $5 \times 10^{5}$ cells/well, and cultured for $48 \mathrm{~h}$ to reach $80 \%$ confluence. The cells were washed twice with PBS. A mixture of individual HMGA2 siRNA $(1 \mu \mathrm{g} / \mathrm{ml})$, transfection medium and Lipofectamine 2000 was added. After $30 \mathrm{~min}, 500 \mu 1$ of Opti-MEM (cat. no. 31985062; Gibco; Thermo Fisher Scientific, Inc.) was added. After $6 \mathrm{~h}$, the transfection medium was removed, and the cells were cultured in RPMI- 1640 medium with $10 \%$ fetal bovine serum. The gene silencing efficiency was confirmed by western blotting and immunocytochemistry $48 \mathrm{~h}$ after the transfection.

Proliferation assays. The cells were seeded at a density of $2 \times 10^{3}$ cells/well in 96-well plates and incubated with RPMI-1640 medium at $37^{\circ} \mathrm{C}$ for 1-7 days. PBS (cat. no. SH30256.01; Hyclone, Logan, UT, USA) was used to dissolve the purple formazan. MTT was added to each well and incubated at $37^{\circ} \mathrm{C}$ for $4 \mathrm{~h}$. The absorbance was measured using a microplate reader with a wavelength of $490 \mathrm{~nm}$. Each experiment was repeated three times.

Transwell migration and invasion assays. The invasion assay was performed as follows. At $48 \mathrm{~h}$ after the transfection, $5 \times 10^{3}$ cells were seeded into the upper compartments of a 24-well Transwell chamber, which was coated with $100 \mu 1$ Matrigel $(200 \mu \mathrm{g} / \mathrm{ml}$; cat. no. 356234; BD Biosciences, Franklin Lakes, NJ, USA). The medium contained with $20 \%$ FBS was added into the lower compartment. After $24 \mathrm{~h}$ incubation, the filter membrane was washed with PBS. The cells were stained with $0.05 \%$ crystal violet and fixed with $10 \%$ formaldehyde. The cells in five random fields of the membrane were counted with microscope. Cell migration assays were performed according to the same protocol, except without Matrigel-coating.
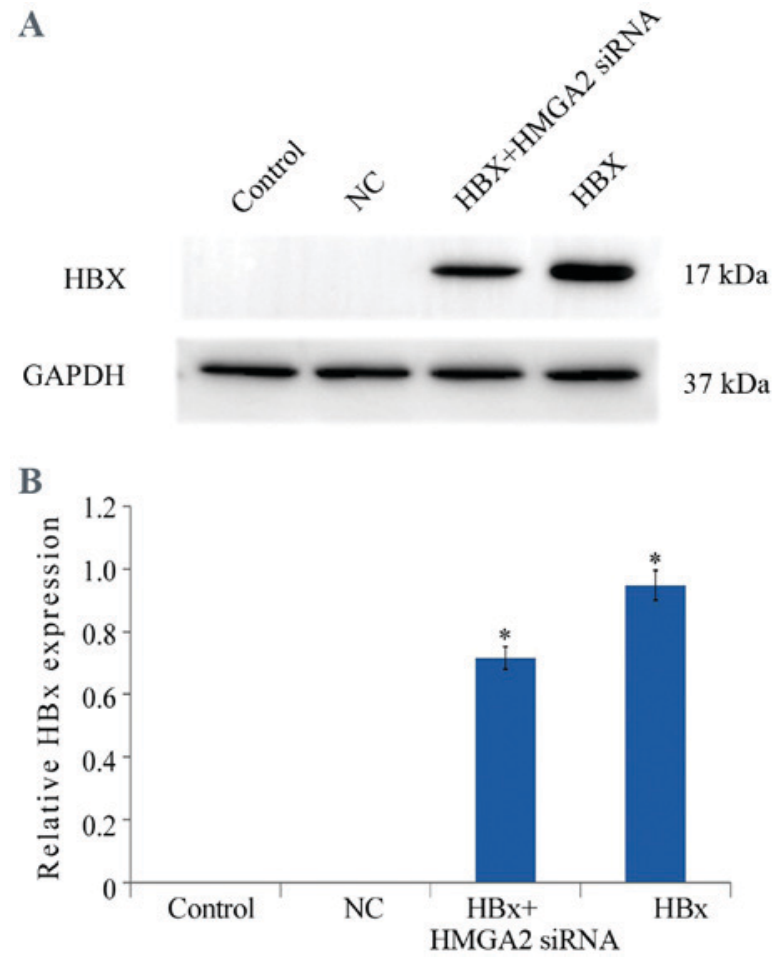

Figure 1. Western blot analysis of HBx in the HCCLM3 cells. GAPDH was used as a loading control. (A) Representative blot and (B) quantification. ${ }^{*} \mathrm{P}<0.05$ vs. control and NC groups. HBX, HBV X protein; siRNA, small interfering RNA; HMGA2, high mobility group AT-hook 2; NC, negative control.

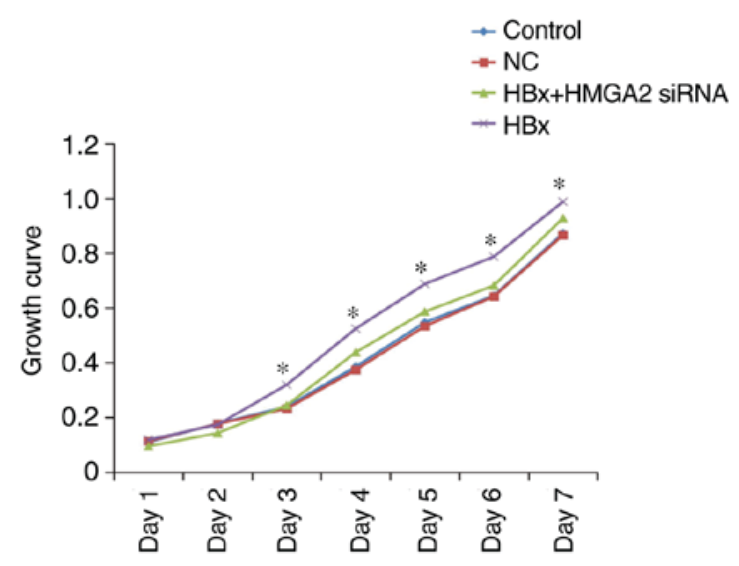

Figure 2. MTT assay of the HCCLM3 cell line. "P<0.05 vs. control and NC groups. HBX, HBV X protein; siRNA, small interfering RNA; HMGA2, high mobility group AT-hook 2; NC, negative control.

Scratch wound-healing assays. A total of $1 \times 10^{6}$ cells were seeded into 6-well tissue culture plates and grown to reach $80 \%$ confluency. A linear wound was created in the cell surface using a 10- $\mu 1$ sterilized pipette tip. The wounded cell layers were washed twice with PBS. The migrated distance at the wound space was measured at 0,24 and $48 \mathrm{~h}$ in five random microscopic fields at magnification, $x 40$ under an Olympus BX53 microscope (Olympus Corporation, Tokyo, Japan). Each independent experiment was repeated three times.

Immunofluorescence. A total of $2 \times 10^{5}$ cells were seeded and cultured onto glass cover slips in 24 -well plates. Then, they 
A

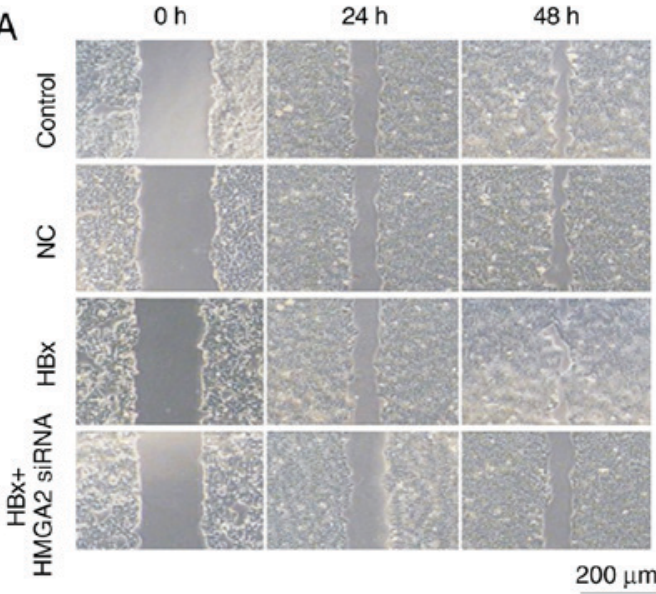

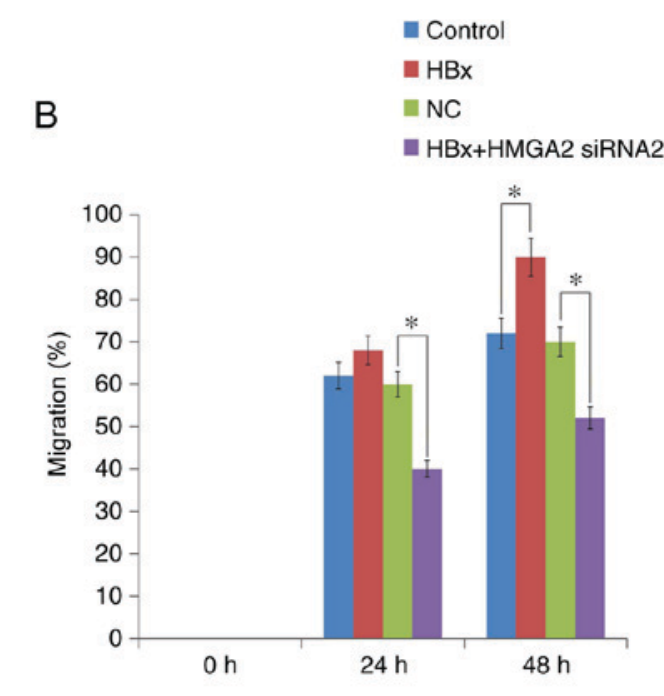

a $\mathrm{HBx}$

$B$

Figure 3. Migratory ability of the HCCLM3 cell line with HBx overexpression or HMGA2 inhibition was determined using a wound-healing assay. (A) Representative image of wound-healing assay and (B) quantification. Wound closure was assessed at 0,24 and $48 \mathrm{~h}$ after wounding (magnification, $\mathrm{x} 40$ ). *P<0.05. HBX, HBV X protein; siRNA, small interfering RNA; HMGA2, high mobility group AT-hook 2; NC, negative control.

were fixed in $4 \%$ paraformaldehyde for $20 \mathrm{~min}$ at $37^{\circ} \mathrm{C}$, and incubated with a primary antibody against HMGA2 (rabbit anti-human; 1:100; cat. no. ab97276; Abcam, Cambridge, UK) overnight at $4^{\circ} \mathrm{C}$. Next, the slides were incubated with an Alexa Fluor 594-conjugated secondary antibody (anti-rabbit immunoglobulin G; 1:500; cat. no. 7074; CST, Boston, USA) for $1 \mathrm{~h}$ at room temperature. Lastly, the cells were stained with DAPI at $37^{\circ} \mathrm{C}$ for $5 \mathrm{~min}$ and observed using fluorescence microscopy at $\mathrm{x} 200$ magnification.

Western blot analysis. The protein was extracted from the cells with 10\% MSDS (Sigma-Aldrich; Merck KGaA, Darmstadt, Germany) and the protein quantity was determined using a bicinchoninic acid assay. The mass of the proteins loaded in per lane was $80 \mu \mathrm{g}$. The proteins were separated using $10 \%$ SDS-PAGE, and then transferred onto polyvinylidene fluoride membranes. The membranes were blocked in 5\% bovine serum albumin (cat. no. 10099141; Gibco; Thermo Fisher Scientific, Inc.) at $37^{\circ} \mathrm{C}$ for $1 \mathrm{~h}$, and washed with TBST buffer three times. The membranes were exposed to primary rabbit anti-human HMGA2 antibodies (cat. no. ab97276; Abcam, San Francisco, USA), HBx (cat. no. ab2741; Abcam, Cambridge, UK), E-cadherin, Vimentin, N-cadherin (all 1:500; Santa Cruz Biotechnology, Inc., Dallas, TX, USA) and GAPDH (1:3,000, ProteinTech Group, Inc., Chicago, IL, USA) overnight at $4^{\circ} \mathrm{C}$. The membranes were washed with TBST three times, and incubated with secondary antibody (anti-rabbit Immunoglobulin G; 1:2,000; cat. no. 7074; CST, Boston, USA) for $2 \mathrm{~h}$ at room temperature, and washed with TBST three times. The bands were visualized with the WEST ZOL Plus system and quantified using ImageJ software (version 1.44P; National Institutes of Health, Bethesda, MA, USA).

Statistical analysis. Statistical analysis was performed using the SPSS version 18.0 (SPSS, Inc., Chicago, IL, USA). Data are expressed as the mean \pm standard deviation of three independent experiments, and were analyzed using the Student's t-test or one-way analysis of variance with the Least Significant
Difference (LSD) post hoc test to determine the different between two groups. $\mathrm{P}<0.05$ was considered to indicate a statistically significant difference.

\section{Results}

$H B x$ promotes proliferation, migration, invasion, and EMT of HCC cells. In order to investigate the effect of HBx on the proliferation of HCC cells, HCCLM3 cells were transfected with the HBx-expressing (pcDNA3.1-HBx) and control vectors (NC). The transfection efficiency was confirmed with western blotting (Fig. 1). MTT analysis demonstrated that the proliferation of HCCLM3 cells transfected with pcDNA3.1-HBx vectors was significantly promoted when compared with the control cells (Fig. 2).

Then, Transwell migration and Matrigel invasion assays were performed to determine the roles of $\mathrm{HBx}$ in $\mathrm{HCC}$ metastasis. The results revealed that HCCLM3 cells transfected with HBx-expressed vectors significantly increased the migratory and invasive capacities compared with empty vector-transfected control cells (Figs. 3 and 4). In addition, Western blot analysis demonstrated that the upregulation of HBx resulted in a significant increase in the epithelial cell marker E-cadherin expression and a decrease in the mesenchymal cell marker Vimentin protein expression in HCCLM3 cells (Fig. 5).

$H B x$ promotes the expression of HMGA2 in HCC cells. To explore the mechanism of HBx on HCC metastasis, the influence of HBx on expression of HMGA2 in HCCLM3 cells was evaluated. Western blotting analyzed indicated that HMGA2 expression was significantly upregulated in HCCLM3 cells transfected with pcDNA3.1-HBx vectors at $48 \mathrm{~h}$ (Fig. 5). Fluorescent microscopy revealed that HBx-transfected HCCLM3 cells had markedly increased HMGA2 expression in the nucleus (Fig. 6). These results suggest that HBx may enhance the metastasis of HCCLM3 cells through HMGA2. 
A

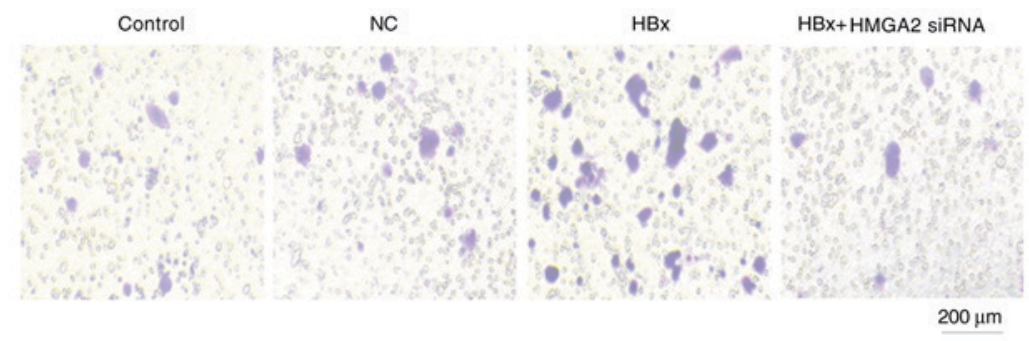

B

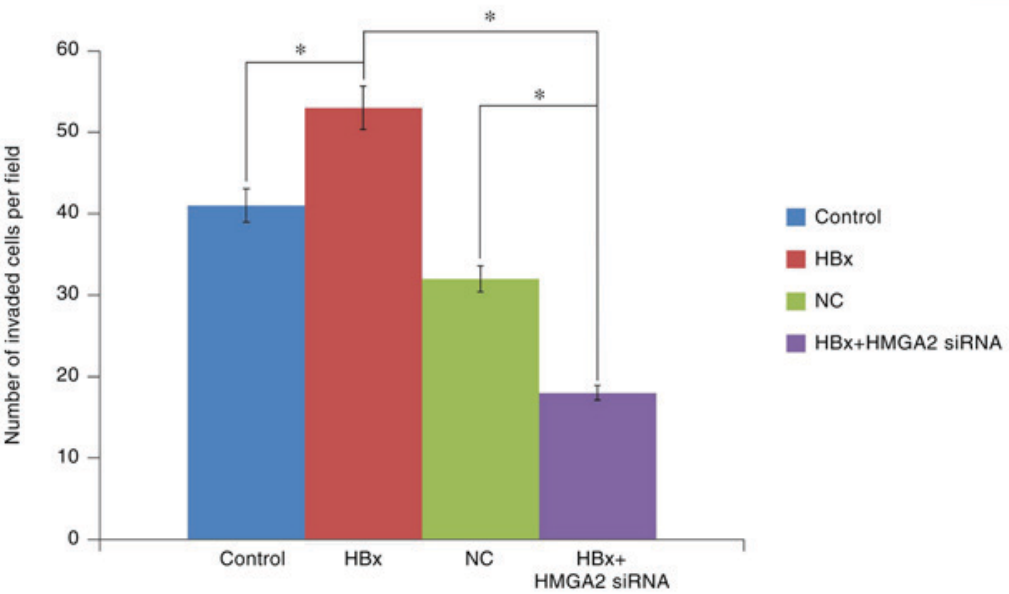

Figure 4. The effect of HBx overexpression and HMGA2 suppression on HCCLM3 cell invasive potential was assessed by the Matrigel invasion assay. (A) Representative image of Matrigel invasion assay and (B) quantification. The number of cells that invaded the basal side of the Matrigel-coated Transwell inserts were counted at $24 \mathrm{~h}$ (magnification, x100). ${ }^{*} \mathrm{P}<0.05$. HBX, HBV X protein; siRNA, small interfering RNA; HMGA2, high mobility group AT-hook 2; NC, negative control.

HMGA2 silencing restrains EMT and metastasis of HCCLM3 cells induced by HBX. To further confirm that HMGA2 was a target of $\mathrm{HBx}$, the effect of HMGA2 knockdown on HBx-induced HCC metastasis was examined. Transwell invasion and scratch wound-healing assays showed that the migratory and invasive capacities of HBx-expressed HCCLM3 cells were significantly inhibited by HMGA2 silencing (Figs. 3 and 4). Furthermore, western blotting analysis demonstrated that HMGA2-siRNA significantly inhibited the expression of E-cadherin and increased in Vimentin protein expression, reversing the EMT in HBx-expressed HCCLM3 cells (Fig. 5). Collectively, these data indicated that HBx enhanced the invasiveness and migration capacities of HCC cells via HMGB2, HMGA2 knockdown was an effective strategy to prevent HBx-induced HCC progression.

\section{Discussion}

HBx is a risk factor for hepatocarcinogenesis and has been implicated in HCC progression. Previous studies reported HBx promoted the proliferation of HCC cells $(5,13)$. HBx also disrupts intercellular adhesion, and induces EMT, invasion, migration and metastasis in $\operatorname{HCC}(13,14)$. In the present study, it was demonstrated that HBx markedly upregulated the expression of the epithelial cell marker E-cadherin and downregulated the expression of the mesenchymal cell marker Vimentin in HCCLM3 cells. In addition, the overexpression of HBx in HCCLM3 cells resulted in significantly increased migratory and invasive capacities compared with the control cells. These data indicated that HBx promoted the EMT, migration and invasion capabilities in HCC.
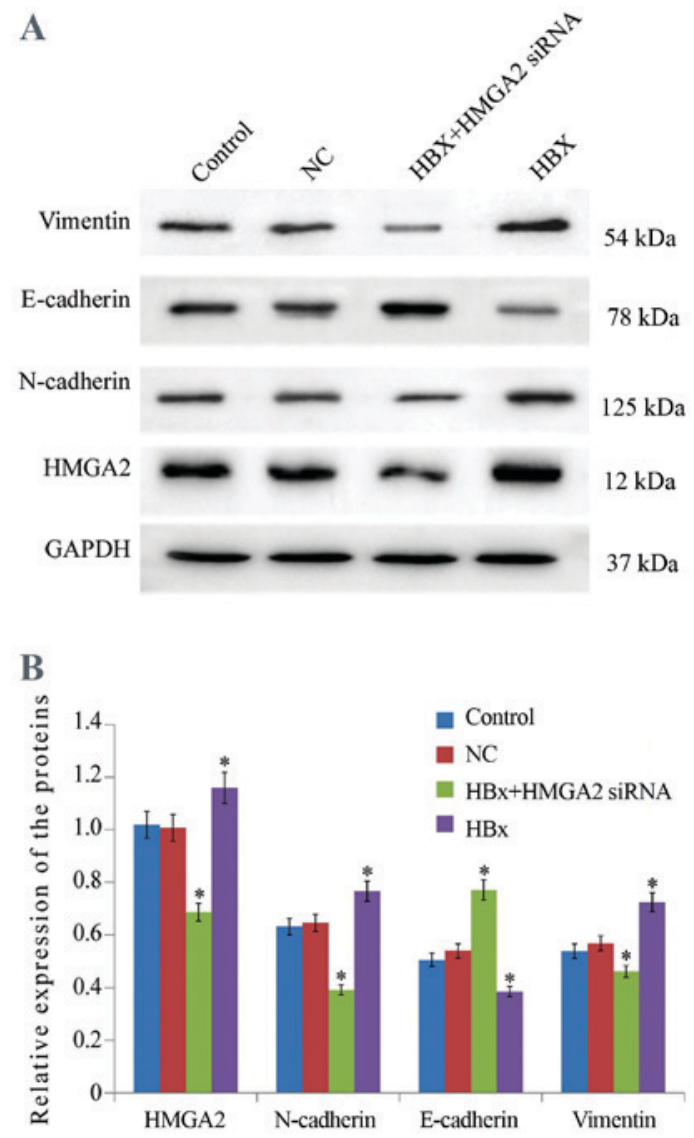

Figure 5. Western blot analysis of HMGA2, E-cadherin, Vimentin and $\mathrm{N}$-cadherin in HCCLM3 cells. (A) Representative blot and (B) quantification. GAPDH was used as a loading control, vs. control and NC groups. ${ }^{*} \mathrm{P}<0.05$. HBX, HBV X protein; siRNA, small interfering RNA; HMGA2, high mobility group AT-hook 2; NC, negative control. 

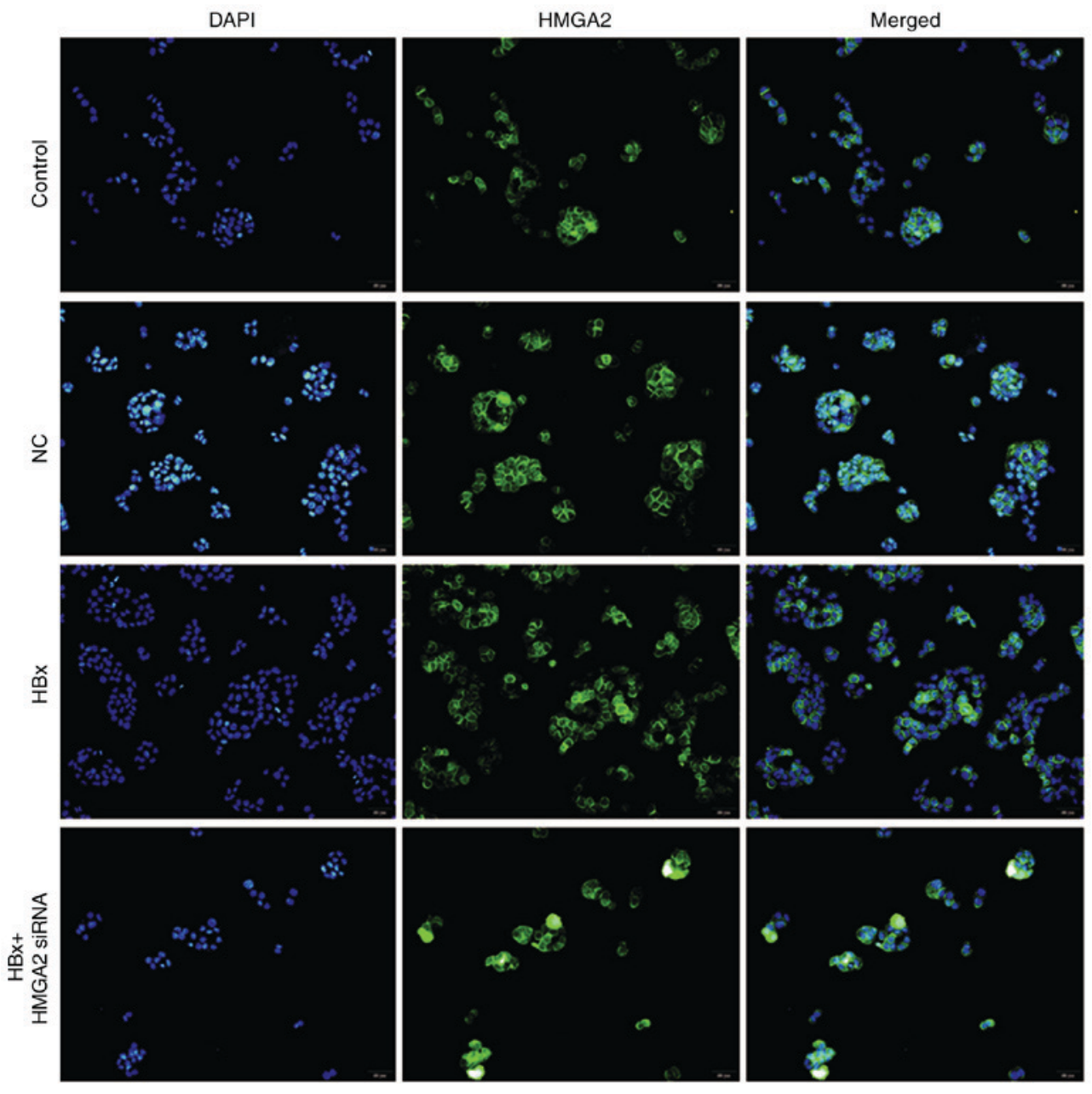

$200 \mu \mathrm{m}$

Figure 6. Immunofluorescence staining of HMGA2 in HCCLM3 cell line was observed by fluorescent microscopy (magnification, x200). HMGA2 located in the nucleus of the cells. HBX, HBV X protein; siRNA, small interfering RNA; HMGA2, high mobility group AT-hook 2; NC, negative control.

Previous studies have reported that HBx promotes HCC metastasis through differing mechanisms. Jin et al (18) reported that HBx promoted HCC cell metastasis and induced EMT by mediating long noncoding RNA, ZEB2 antisense RNA 1. Hou et al (19) reported that HBx promoted HCC cell invasion and migration through the $\mathrm{HBx}$-metastasis associated lung adenocarcinoma transcript 1 (non-protein coding)/latent transforming growth factor $\beta$ binding protein 3 signaling axis. Chen et al (6) demonstrated that HBx overexpression induced the secretion of high-mobility group box 1 to promote the invasion and metastasis of HCC in an autocrine/paracrine manner. In current study, it was revealed that HMGA2 knockdown inhibited HBx-induced EMT and metastasis in HCCLM 3 cells. The HMGA2 protein overexpression has been reported to be associated with metastasis in HCC (20-22). Ou et al (20) demonstrated that HMGA2 promoted HCC cell proliferation and invasion via the $\mathrm{Wnt} / \beta$-catenin signaling pathway. Luo et al (21) reported that HMGA2 expression was significantly associated with the expression of EMT markers, whereby HMGA2 induced EMT by upregulating the expression of Twist and Snail in HCC cell lines. The results suggest that HBx promotes HCC progression at least in part through targeting HMGA2, HMGA2 is an effective target to prevent HBx-induced HCC metastasis.
Previously, certain studies have reported that HBx upregulated transforming growth factor- $\beta 1$ (TGF $\beta 1$ ) expression $(23,24)$. TGF- $\beta$ was also a vital cytokine to activate HMGA 2 and induce EMT in HCC (25). Increased TGF- $\beta$ levels may upregulate HMGA2 expression, which in turn promotes the EMT, invasion and migration capabilities HCC. The data suggested that HBx activated HMGA2 through TGF- $\beta$. However, further studies are required to confirm this hypothesis.

There are certain limitations to the present study. Firstly, the use of different experimental systems and HBx expression levels may result in differences in the effects of $\mathrm{HBx}$ on cellular signal transduction pathways and influence HCC metastasis. Secondly, since HBx has been identified to serve a vital function in the regulation of chronic liver inflammation and the liver tumor microenvironment (26), further study is required to clarify the interaction between HBx and HMGA2 under the specific liver tumor microenvironment. Finally, it has been reported that overexpression of HBx in hepatocellular carcinoma cells may induce the secretion of high-mobility group box 1 (HMGB1) to promote invasion and metastasis of HCC in an autocrine/paracrine manner (6). However, the interactions between HMGB1 and HMGB2 in HBx-induced HCC metastasis remain largely unclear. 
In conclusion, in spite of the aforementioned limitations in the current study, an important role of the HBx-induced HMGA2 signaling pathway in regulating EMT was identified, which subsequently promotes the invasive and migratory invasive capacities of HCC. The present study suggests that HMGA2 may serve as a potential therapeutic target for the treatment of patients with HBV-associated HCC.

\section{Acknowledgements}

The authors would like to thank Dr Linbo Gao for having reviewed the language of the manuscript prior to submission.

\section{Funding}

The present study was supported in part by grants from the Projects of National Natural Science Foundation of China (grant no. 81560497) and Applied Basic Research Projects-Joint special project of Yunnan Province (grant no. 2015FB073).

\section{Availability of data and materials}

All analyzed data and materials in this study are included in this published article.

\section{Authors' contributions}

YZ designed the study. QY, JSL, YYW and WMS completed the experiment. YZ drafted the manuscript. All authors have approved the final version.

\section{Ethics approval and consent to participate}

Not applicable.

\section{Patient consent for publication}

Not applicable.

\section{Competing interests}

The authors declare no competing interests.

\section{References}

1. Akamatsu N, Cillo U, Cucchetti A, Donadon M, Pinna AD, Torzilli $\mathrm{G}$ and Kokudo N: Surgery and hepatocellular carcinoma. Liver Cancer 6: 44-50, 2016.

2. Tseng TC and Kao JH: HBV markers for HCC prediction: Three heads are better than two? J Hepatol 67: 203-204, 2017.

3. Zhou SJ, Deng YL, Liang HF, Jaoude JC and Liu FY: Hepatitis $B$ virus $X$ protein promotes CREB-mediated activation of miR-3188 and Notch signaling in hepatocellular carcinoma. Cell Death Differ 24: 1577-1587, 2017.

4. Yang ST, Yen CJ, Lai CH, Lin YJ, Chang KC, Lee JC, Liu YW, Chang-Liao PY, Hsu LS, Chang WC, et al: SUMOylated CPAP is required for IKK-mediated NF- $\kappa \mathrm{B}$ activation and enhances HBx-induced NF- $\kappa B$ signaling in HCC. J Hepatol 58: 1157-1164, 2013.

5. Zhu M, Li W, Lu Y, Dong X, Lin B, Chen Y, Zhang X, Guo J and $\mathrm{Li} \mathrm{M}$ : HBx drives alpha fetoprotein expression to promote initiation of liver cancer stem cells through activating PI3K/AKT signal pathway. Int J Cancer 140: 1346-1355, 2017.

6. Chen S, Dong Z, Yang P, Wang X, Jin G, Yu H, Chen L, Li L, Tang L, Bai S, et al: Hepatitis B virus X protein stimulates high mobility group box 1 secretion and enhances hepatocellular carcinoma metastasis. Cancer Lett 394: 22-32, 2017.
7. Fusco A and Fedele M: Roles of HMGA proteins in cancer. Nat Rev Cancer 7: 899-910, 2007.

8. Franco R, Esposito F, Fedele M, Liguori G, Pierantoni GM, Botti G, Tramontano D, Fusco A and Chieffi P: Detection of high-mobility group proteins A1 and A2 represents a valid diagnostic marker in post-pubertal testicular germ cell tumours. J Pathol 214: 58-64, 2008.

9. Sun M, Gomes S, Chen P, Frankenberger CA, Sankarasharma D, Chung $\mathrm{CH}$, Chada KK and Rosner MR: RKIP and HMGA2 regulate breast tumor survival and metastasis through lysyl oxidase and syndecan-2. Oncogene 33: 3528-3537, 2014.

10. Piton N, Angot É, Marguet F and Sabourin JC: HMGA2 immunostaining is a straightforward technique which helps to distinguish pulmonary fat-forming lesions from normal adipose tissue in small biopsies: A retrospective observational study about a series of 13 lung biopsies. Diagn Pathol 12: 21, 2017.

11. Wu J,Zhang S, Shan J, Hu Z, Liu X, Chen L, Ren X, Yao L, Sheng H, $\mathrm{Li} \mathrm{L}$, et al: Elevated HMGA2 expression is associated with cancer aggressiveness and predicts poor outcome in breast cancer. Cancer Lett 376: 284-292, 2016.

12. Dong J, Wang R, Ren G, Li X, Wang J, Sun Y, Liang J, Nie Y, Wu K, Feng B, et al: HMGA2-FOXL2 axis regulates metastases and epithelial-to-mesenchymal transition of chemoresistant gastric cancer. Clin Cancer Res 23: 3461-3473, 2017.

13. Chen Z, Tang J, Cai X, Huang Y, Gao Q, Liang L, Tian L, Yang Y, Zheng Y, Hu Y and Tang N: HBx mutations promote hepatoma cell migration through the Wnt/ $/$-catenin signaling pathway. Cancer Sci 107: 1380-1389, 2016.

14. Ha HL, Kwon T, Bak IS, Erikson RL, Kim BY and Yu DY: IGF-II induced by hepatitis B virus X protein regulates EMT via SUMO mediated loss of E-cadherin in mice. Oncotarget 7: 56944-56957, 2016.

15. Agostini A, Panagopoulos I, Andersen HK, Johannesen LE, Davidson B, Tropé CG, Heim S and Micci F: HMGA2 expression pattern and TERT mutations in tumors of the vulva. Oncol Rep 33: 2675-2680, 2015.

16. Li Y, Zhao Z, Xu C, Zhou Z, Zhu Z and You T: HMGA2 induces transcription factor Slug expression to promote epithelial-to-mesenchymal transition and contributes to colon cancer progression. Cancer Lett 355: 130-140, 2014.

17. Guo SP, Wang WL, Zhai YQ and Zhao YL: Expression of nuclear factor-kappa B in hepatocellular carcinoma and its relation with the X protein of hepatitis B virus. World J Gastroenterol 7: 340-344, 2001.

18. Jin Y, Wu D, Yang W, Weng M, Li Y, Wang X, Zhang X, Jin X and Wang T: Hepatitis B virus x protein induces epithelial-mesenchymal transition of hepatocellular carcinoma cells by regulating long non-coding RNA. Virol J 14: 238, 2017.

19. Hou Z, Xu X, Fu X, Tao S, Zhou J, Liu S and Tan D: HBx-related long non-coding RNA MALAT1 promotes cell metastasis via up-regulating LTBP3 in hepatocellular carcinoma. Am J Cancer Res 7: 845-856, 2017

20. Ou W, Lv J, Zou X, Yao Y, Wu J, Yang J, Wang Z and Ma Y: Propofol inhibits hepatocellular carcinoma growth and invasion through the HMGA2-mediated Wnt/ $\beta$-catenin pathway. Exp Ther Med 13: 2501-2506, 2017.

21. Luo Y,LiWandLiaoH:HMGA2 inducesepithelial-to-mesenchymal transition in human hepatocellular carcinoma cells. Oncol Lett 5: 1353-1356, 2013.

22. Wang Y, Chen F, Zhao M, Yang Z, Li J, Zhang S, Zhang W, Ye L and Zhang X: The long noncoding RNA HULC promotes liver cancer by increasing the expression of the HMGA2 oncogene via sequestration of the microRNA-186. J Biol Chem 292: 15395-15407, 2017.

23. Liu Y, Xu Y, Ma H, Wang B, Xu L, Zhang H, Song X, Gao L, Liang $X$ and Ma C: Hepatitis B virus X protein amplifies TGF- $\beta$ promotion on HCC motility through down-regulating PPM1a. Oncotarget 7: 33125-33123, 2016.

24. Murata M, Matsuzaki K, Yoshida K, Sekimoto G, Tahashi Y, Mori S, Uemura Y, Sakaida N, Fujisawa J, Seki T, et al: Hepatitis $\mathrm{B}$ virus $\mathrm{X}$ protein shifts human hepatic transforming growth factor (TGF)-beta signaling from tumor suppression to oncogenesis in early chronic hepatitis B. Hepatology 49: 1203-1217, 2009.

25. Morishita A, Zaidi MR, Mitoro A, Sankarasharma D, Szabolcs M, Okada Y, D'Armiento J and Chada K: HMGA2 is a driver of tumor metastasis. Cancer Res 73: 4289-4299, 2013.

26. Fu S, Zhou RR, Li N, Huang Y and Fan XG: Hepatitis B virus X protein in liver tumor microenvironment. Tumour Biol: Sep 23, 2016 (Epub ahead of print).

This work is licensed under a Creative Commons Attribution-NonCommercial-NoDerivatives 4.0 International (CC BY-NC-ND 4.0) License. 